\title{
Optically Sensing for Thyroid Profile Hormones in Blood
}

\author{
Rawia Shatti ${ }^{1}$, Layla M.H. Al-ameri ${ }^{2}$ \\ ${ }^{1}$ Scholar Researcher, ${ }^{2}$ Lecturer, Institute of Laser for Postgraduate Studies, University of Baghdad, Iraq
}

\begin{abstract}
Optical biosensors present great characteristics compared to traditional analytical techniques because they allow direct, realistic, and label-free detection of many biological and chemical materials. Thyroid hormones are created by the thyroid gland, which is found in the abdomen of the thyroid cartilage Thyroid hormone governs metabolic processes necessary for normal outgrowth and evolution in addition to regulating metabolism in adults. It is established that the condition of the thyroid hormone is related to body weight and energy disbursement. This work using a single-mode tapered fiber biosensor to detect changes in the level of thyroid hormone by measurement of the intensity changing with the concentration of hormone by using a diode laser $(450 \mathrm{~nm})$ wavelength as an optical source, the single-mode tapered fiber as the sensor connected with ocean (HR 2000) spectrum analyzer by an adapter and finally connected to the pc to show the result. The lowest intensity of transmitted light recorded with a higher concentration of the hormone because of the high matching and specific selection of laser wavelength with the structure of the thyroid hormone in which give the specific amount of the hormone present in the sample. Thyroid hormone can be detected by using a laser biosensor. The higher concentration of thyroid hormone referred to the lowest intensity of light.
\end{abstract}

Keywords: Optically sensing, thyroid profile, hormones.

\section{Introduction}

Thyroid hormone governs are metabolic processes necessary for normal outgrowth and evolution in addition to regulating metabolism in adults. It is established that the condition of the thyroid hormone is related to body weight and energy disbursement. Hyperthyroidism, promotes excessive metabolic status characterized by increased energy consumption during rest, weight loss, low cholesterol levels, increased fat decomposition, and glucose formation. On the contrary, hypothyroidism, low levels of thyroid hormone, is associated with a lack of metabolism which is characterized by low resting energy consumption, weight gain, increased cholesterol levels, low-fat degradation, and reduced sugar development. TH catalyzes both lip formation and fat decomposition, although TH levels are high, the net effect is fat lack ${ }^{[1]}$.

\section{Corresponding Author: \\ Rawia Shatti}

Scholar Researcher, Institute of Laser for Postgraduate Studies, University of Baghdad, Iraq

e-mail: rawiashatii@gmail.com
The core volume of single-mode fiber is little. The size of the core (diameter) is usually about 8 to 10 micrometers $(\mu \mathrm{m})$. The core of fiber of that volume is allowed only to place the platform or minimum to spread around the wavelength of $1300 \mathrm{~nm}(\mathrm{~nm})$. Single-mode fibers are spread in only one mode because the base size approaches the operational wavelength $(\lambda)$. This is accomplished using lasers as a source of light ${ }^{[2]}$. The primary refraction coefficient can be referred to as $n$, which is larger than the $\mathrm{n}$ cladding refraction coefficient. The little diameter of the nucleus lets the transfer of one position of the light. No dispersion, no degeneration of the signal transmitted through the fiber, low attenuation due to the number of waves spreading along with the core of the fiber giving the signal ability to spread over long distances and faster. As shown in figure (1)

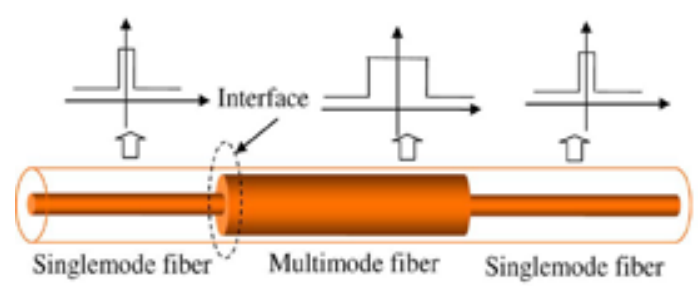

Figure (1): Schematic_configuration of (single mode-multimode single mode fiber) structure ${ }^{[4]}$ 
Optical biosensors present great characteristics compared to traditional analytical techniques because they allow direct, realistic, and label-free detection of many biological and chemical materials. Its features include high privacy, sensitivity, little size, and costeffectiveness ${ }^{[5]}$. Biochemical processes play a very important role in medicine, biology, and biotechnology. However, it is very rough to convert biological data directly into an electrical signal, biosensors can transform these signals and biological sensors to this difficulty. In recent years, thanks to improved technologies and devices, the use of these products has increased ${ }^{[6]}$. While sensors have himself group of components: light sources and optical fiber to direct light between them and the optical detectors. So Understanding the principle of the action of different fiber optic sensors, it is important to find out the optical components used [7]. The study aimed to assess of the level of concentrations of thyroid hormones (Thyroid-stimulating (THS) and thyroxin (T4) \& Triiodothyronine (T3)) by using a single-mode optical sensor (SMF) as a new method.

\section{Material and Method}

Sample Collection: Ten $\mathrm{ml}$ was taken from the blood sample of each patient, each sample then centrifuged to get serum and this serum is divided into two parts, each part is five $\mathrm{ml}$, the first part is used for manually detection of (T3 and T4 and TSH) by using Vida's device, the other part $(5 \mathrm{ml})$ is used for detection the three hormone (T3, T4, and TSH) by using a singlemode optical sensor (SMF).

Biological Measurement: The levels of serum thyroid hormone (T3, T4, TSH) were measured by Vida>s device is an automated quantitative test for use on Vida $\mathrm{S}$ family instruments for the quantitative measurement of total thyroid hormone (T3, T4, TSH) in human serum (lithium heparin) using the ELFA technique (EnzymeLinked Fluorescent Assay).

Fabication of SMF: A $(30 \mathrm{~cm})$ long fiber sensor is considered a traditional optical fiber cut a length of about $(2 \mathrm{~cm})$ in the middle of the fibers using a cut Make grooves on each side. The buffer was removed byways of dipping the pieces in $40 \%$ acetone concentration for (30 minutes). Washed with distilled water. to get rid of impurities and clean it well. A part of the fiber is immersed in pure hydrofluoric acid (HF) $40 \%$. to remove the cladding of the fiber, in a (10-minutes) setup and then washed with distilled water. The full fibers $(30 \mathrm{~cm})$, was put in the aplastic device using an adhesive silicone. The ends of the fibers are connected to a transformer device. The terminal tools are connected to optical fibers by optical fiber connectors. Splicing has been used to Join two organized fibers to form an unending optical waveguide. The first end is connected to a laser exporter (blue diode laser) and power supply. Diode laser, (450) nm wavelength, and an output power $<50,000 \mathrm{MW}$ was used. The Laser wavelength matches the absorption peaks of the three thyroid hormones (T3, T4and TSH). So it connected to a stable power supply. This laser was selected depending on the absorption spectra of the hormones which were measured by using a spectrophotometer. Another part is connected it is a spectrum analyzer (ocean HR2000) to get an intensity signal.

Optical Spectrum Analyzer (Ambient Optics HR2000) with accuracy $(0.035 \mathrm{~nm})$ of high wavelength and works at a wavelength of (200-1100 nm), and connects the spectrometer Via a USB port or serial port to a laptop or desktop computer.

Table [1] Thyroid hormone standard concentration and intensity.

\begin{tabular}{|c|c|c|}
\hline Types of thyroid hormone & $\begin{array}{c}\text { Standard Concentration n mol/L } \\
\text { (Measured by Vida's Device) }\end{array}$ & $\begin{array}{c}\text { Intensity in single mode fiber (SM1) } \\
\text { biosensor }\end{array}$ \\
\hline T3 & $2 \mathrm{ml}$ & 2803.71 \\
\hline $\mathrm{T} 4$ & $4 \mathrm{ml}$ & 2365.13 \\
\hline $\mathrm{TSH}$ & $2 \mathrm{ml}$ & 2446.87 \\
\hline
\end{tabular}


Table [2]: Samples concentration and intensity.

\begin{tabular}{|c|c|c|c|c|c|c|}
\hline \multicolumn{2}{|c|}{ TSH } & \multicolumn{2}{|c|}{ T4 } & \multicolumn{2}{|c|}{ T3 } & \multirow[b]{2}{*}{ Numbers } \\
\hline $\begin{array}{l}\text { Intensity in au } \\
\text { single mode } \\
\text { fiber (SMF) } \\
\text { biosensor }\end{array}$ & $\begin{array}{c}\text { Concentration } \\
\text { of TSH n mol/L } \\
\text { (Measured by } \\
\text { Vida's Device) }\end{array}$ & $\begin{array}{l}\text { Intensity au) } \\
\text { in) single mode } \\
\text { fiber (SMF) } \\
\text { biosensor }\end{array}$ & $\begin{array}{l}\text { Concentration } \\
\text { of T4 } \mathrm{n} \mathrm{mol} / \mathrm{L} \\
\text { (Measured by } \\
\text { Vida's Device) }\end{array}$ & $\begin{array}{l}\text { Intensity au } \\
\text { in single mode } \\
\text { fiber (SMF) } \\
\text { biosensor }\end{array}$ & $\begin{array}{l}\text { Concentration } \\
\text { of T3 n mol/L } \\
\text { (Measured by } \\
\text { Vida's Device) }\end{array}$ & \\
\hline 1463.0 & $>60.0$ & 1497.86 & 175 & 1526.86 & 4.3 & 1 \\
\hline 1776.0 & 18.5 & 1525 & 172 & 1556.57 & 3.3 & 2 \\
\hline 1785.71 & 16.6 & 1622.57 & 129 & 1652.57 & 3.2 & 3 \\
\hline 1797.71 & 16.5 & 1665.77 & 128.5 & 1668.57 & 3.0 & 4 \\
\hline 2029.71 & 7.4 & 1814.29 & 123 & 1749.71 & 2.4 & 5 \\
\hline 2172.14 & 4.3 & 2446.29 & 111 & 2464.29 & 2.0 & 6 \\
\hline 2217.14 & 3.0 & 2459.14 & 96 & 2473.71 & 1.9 & 7 \\
\hline 2237.14 & 2.3 & 2464.29 & 92 & 2557.71 & 1.7 & 8 \\
\hline 2276.43 & 1,0 & 2508.86 & 88 & 2568.86 & 1.5 & 9 \\
\hline 2356.43 & 0.7 & 2588.57 & 62 & 2607.43 & 1.3 & 10 \\
\hline 2780.57 & 0.24 & 2740.29 & 56.0 & 2654.57 & 0.9 & 11 \\
\hline 2782.29 & 0.21 & 2749.71 & 53.7 & 2664.86 & 0.8 & 12 \\
\hline 2791.71 & 0.10 & 2802.86 & 47.7 & 2674.29 & 0.7 & 13 \\
\hline 2797.0 & 0.09 & 2839.71 & 44.9 & 2714.57 & $<0.4$ & 14 \\
\hline 2811.43 & $<0.05$ & 3280 & 16.0 & 2785.71 & $<0.4$ & 15 \\
\hline
\end{tabular}

Assess variation in thyroid hormone level by using a single- mode fiber optical biosensor. As shown in figures $(2,3,4)$

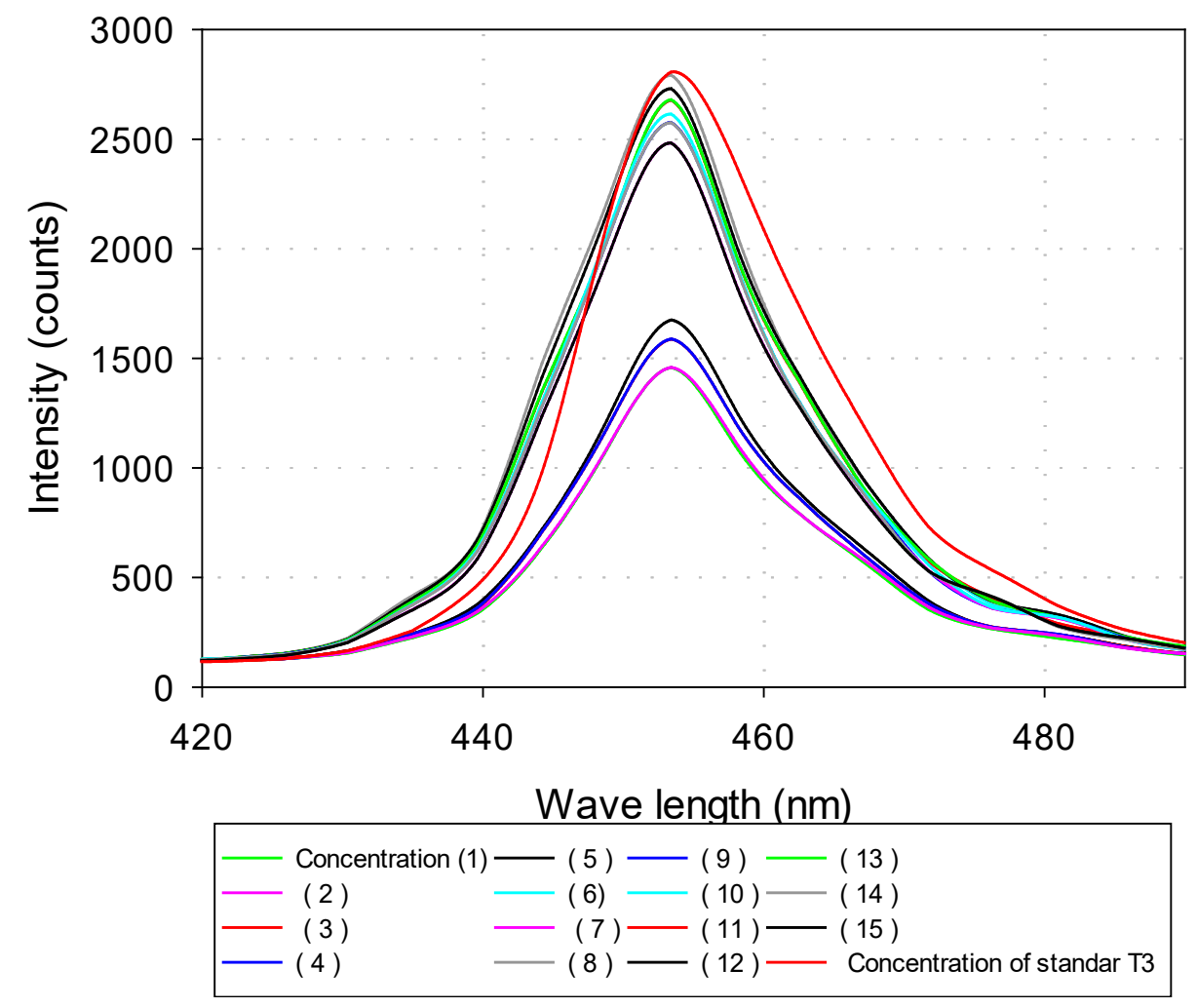

Figure (2) T3 specimen and stander Intensity peak in SMF 


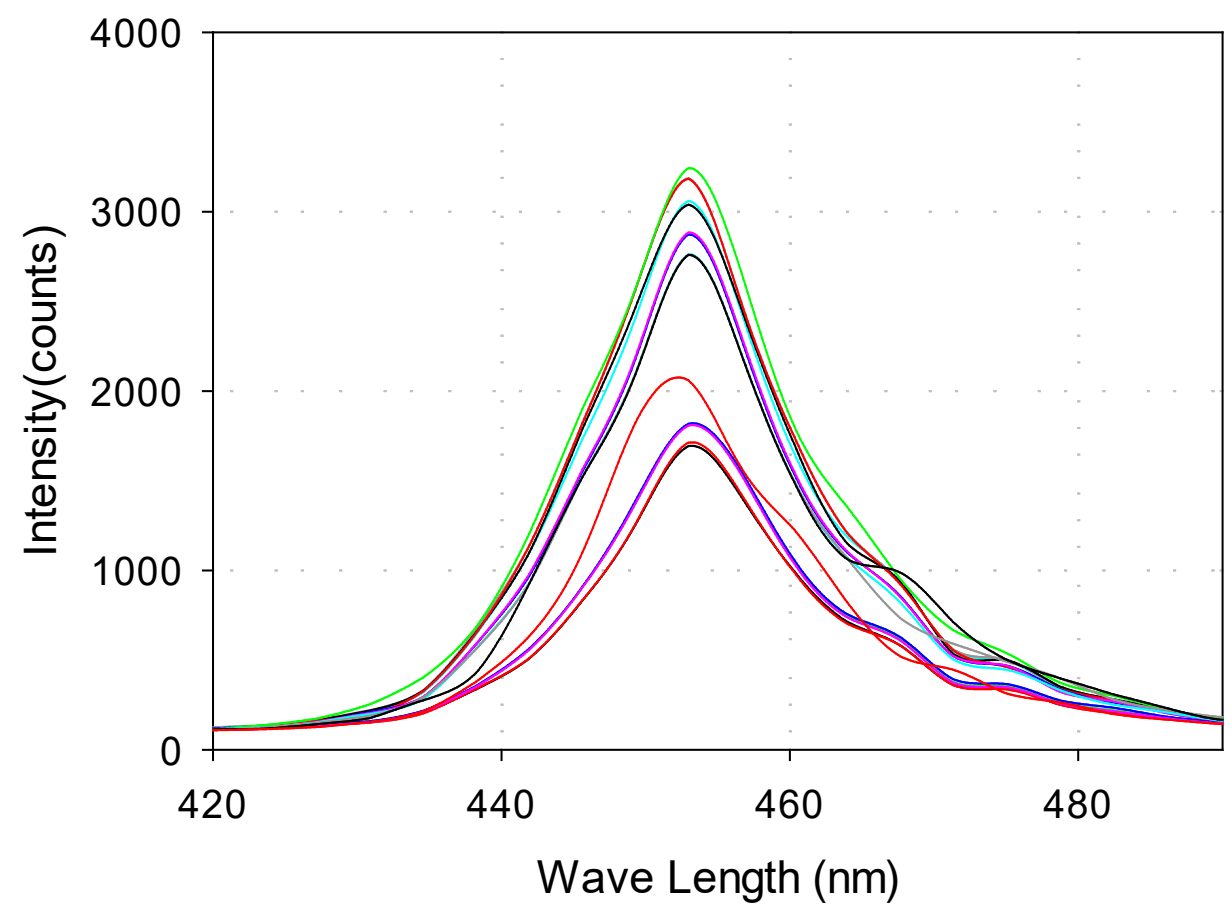

\begin{tabular}{|c|c|c|}
\hline $\begin{array}{l}\text { Concentration ( } 1 \text { ) } \\
(2) \\
(3) \\
(4)\end{array}$ & 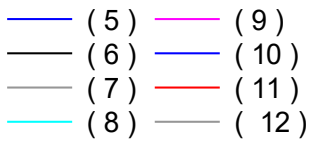 & $\begin{array}{l}\bar{L}(13) \\
\overline{-}(14) \\
-(15) \\
\text { Concentration of standar T4 }\end{array}$ \\
\hline
\end{tabular}

Figure (3) T4 specimen and stander Intensity peak in SMF

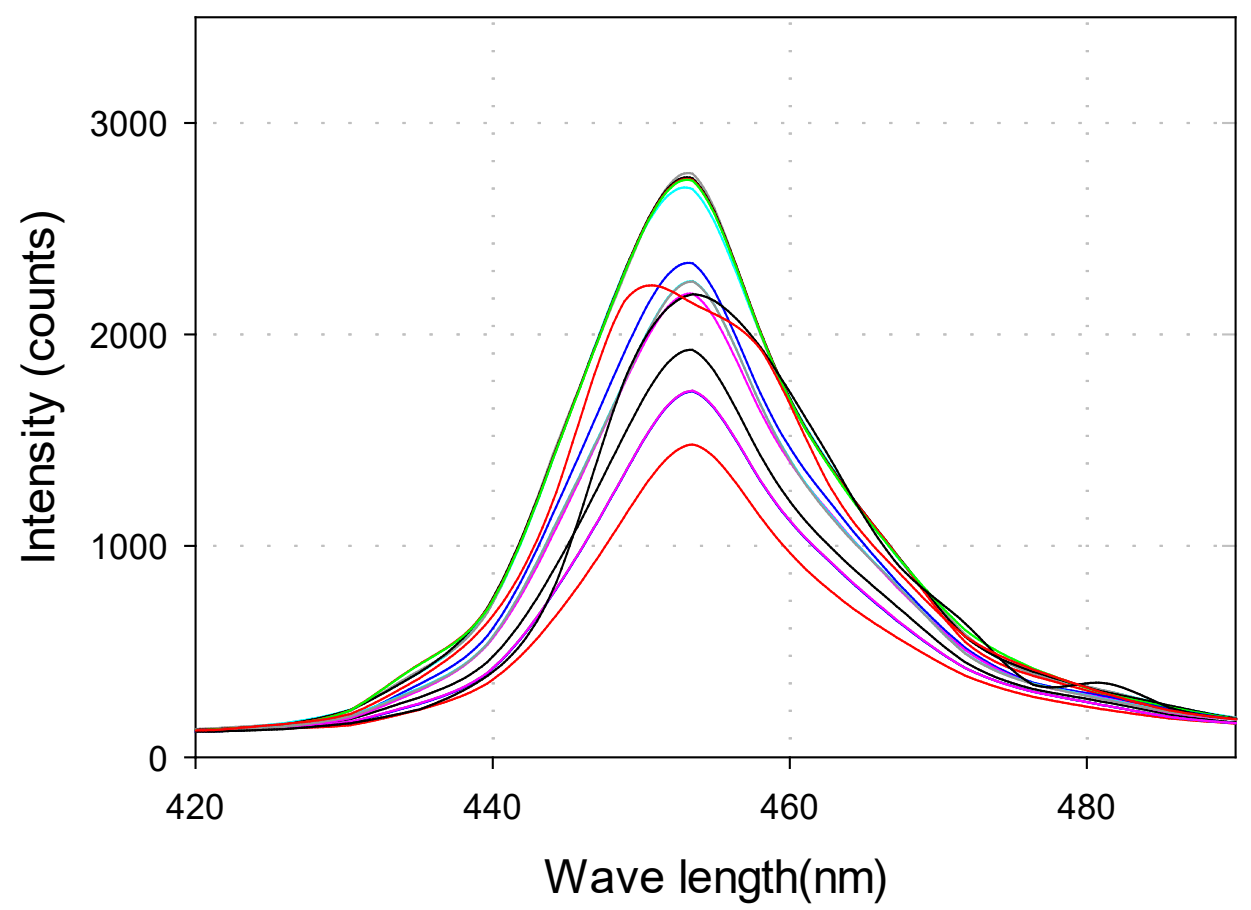

\begin{tabular}{|c|c|c|c|}
\hline $\begin{array}{l}- \text { Concentration( } 1 \text { ) } \\
-(2) \\
-(3) \\
\text { (4) }\end{array}$ & $\begin{array}{r}-(5) \\
-(6) \\
-(7) \\
-(8)\end{array}$ & $\begin{array}{l}\square \\
\square(9) \\
-(10) \\
-(11) \\
-(12)\end{array}$ & $\begin{array}{l}-(13) \\
-(14) \\
-(15) \\
-\quad \text { Concentration of stander TSH }\end{array}$ \\
\hline
\end{tabular}

Figure (4) TSH specimen and stander Intensity peak in SMF 


\section{Results and Discussion}

Tables 1 and 2 show the concentration of the stander of hormone which obtained from the ELFA kit and the concentration of the hormone after using the ELFA technique (Enzyme-Linked Fluorescent Assay). Method and the intensity of each concentration obtained from the optical biosensor. From the result inverse relationship between the concentration and the absorbance intensity, the highest the intensity was in the lowest concentration and that indicates the low amount of thyroid hormone present in the serum and because of the specific absorbance and high matching in the $450 \mathrm{~nm}$ wavelength for thyroid hormone we could know the specific amount of the hormone in the sample by using the Single-Mode Fiber sensor and this agrees with ${ }^{[9]}$ the manufactured one sensor using type of fiber (SMF) for detection some on types of female hormones $(\mathrm{LH})$ by using a diode laser(blue) at a wavelength (450um) as a source of light ${ }^{[8]}$. This agrees with ${ }^{[9]}$ where she manufactured one sensor using Mach-Zehnder interferometer (MZI) for detection of the hemoglobin concentration in human blood was designed by Green laser with a wavelength of $532 \mathrm{~nm}$ and input power of $12.2 \mathrm{nw}$ has been used as a source of light ${ }^{[9]}$.

Conflict of Interest: None

Funding: Self

Ethical Clearance: Not required

\section{Conclusion}

Use a single-mode optical fiber sensor with a laser source $(450 \mathrm{~nm})$ to detect the thyroid hormone level. From the result of the study, we found that the maximum laser intensity indicates the lowest concentration of the hormones. A sensor is most accurate, with a rapid diagnosis, less costly method than the method.

\section{References}

1. Mullur R, Liu YY, Brent GA. Thyroid hormone regulation of metabolism. Physiological reviews. 2014 Apr;94(2):355-82.

2. Perdigão MS, Trovão JP, Alonso JM, Saraiva ES. Large-signal characterization of power inductors in EV bidirectional DC-DC converters focused on core size optimization. IEEE Transactions on Industrial Electronics. 2015 Feb 11;62(5):3042-51.

3. S.M.Dhanshetti, "AdvanceModule Communication System and Specilized Module D th and Other Communication System”, India, 2015.

4. Wang Q, Farrell G, Yan W. Investigation on singlemode-multimode-single-mode fiber structure. Journal of Lightwave Technology. 2008 Mar 3;26(5):512-9.

5. P. Damborský, J. Švitel, and J. Katrlík, “Optical biosensors,” Essays Biochem., 2016. vol. 60, no. 1, pp. 91-100.

6. J. Haus, "Optical Sensors Basics and Applications," 2010. Vol.53, No.1.

7. S. K. Alageedi and L. M. Alameri, "Assessment of Variation In Luteinizing Hormone (LH) Level Among Females By Using Single-Mode Fiber Optical Biosensor (SMF)," 2019. vol. 19, no. 2, pp. 4221-4224.

8. F.F. Rashid, S.J. Belal, L.M. Alameri, and T.S. Mansour, "Human Blood Hemoglobin Concentration," 2019. pp. 27-31. 